\title{
Utilization, Cost, and Pricing Scheme of Compounded Medications for Public Health System Patients: The California Workers' Compensation System, 2011-2013
}

\author{
Tracy Kuo Lin, MSc, PhD; Osama A. Shoair, PhD; Dang Tran, PharmD; and Leslie Wilson, PhD
}

\begin{abstract}
BACKGROUND: Although medically necessary in some cases, there is growing concern that compounded medications are being overprescribed, leading to questions about safety and necessity for high use and cost. Safety concerns regarding compounded medications were highlighted by the 2013 contamination of steroid injections by the New England Compounding Center, which caused serious infections and other injuries to at least 751 patients and resulted in at least 64 patient deaths. A study contributed to our understanding of compounded medication use and cost, finding in a sample of commercially insured population that the average ingredient cost for compounded medication prescriptions was $\$ 710.36$, which is $130 \%$ higher than for noncompounded medication prescriptions. The literature on use and cost of compounded medications in noncommercially insured populations and related regulations, however, is sparse. The California Workers' Compensation System (CAWCS) —-the largest U.S. workers' compensation system and a public health system experiencing high compounded medication costs-provided an opportunity for additional analysis of these issues. Furthermore, CAWCS data on compounded medication use and cost allow for the exploration of alternative pricing mechanisms that may control costs.
\end{abstract}

OBJECTIVES: To (a) examine use, cost, and billing and reimbursement practices for compounded medications in a public health system-CAWCSand (b) evaluate regulations and recommend an alternative pricing mechanism that could control costs in California.

METHODS: Descriptive statistics for use, cost, and reimbursement patterns of all compounded medication prescriptions included in CAWCS's Workers Compensation Information System claims datasets from 2011 to 2013 were determined. This study coded a unique dataset that (a) identified compounded medications at the ingredient level; (b) grouped compounded medications from ingredient level to compounded medications as a whole; and (c) categorized compounded medications into applicable Colorado pricing categories. T-tests assessed if regulation AB 378, which targets compounded medications, was associated with a difference in mean cost. The Colorado pricing scheme was applied to estimate cost and provide recommendations.

RESULTS: Despite the AB 378 requirement for compounded medications to be billed at the ingredient level for reimbursement, $15 \%$ of pharmacy-dispensed and $6 \%$ of physician-dispensed medications were not billed at the ingredient level. For pharmacy-dispensed compounded medications billed at the ingredient level, mean amount paid (SD) per ingredient was $\$ 45.40$ (195.97), and for those medications billed at the single compounded medication level, mean amount paid (SD) per medication was $\$ 95.20$ (326.33) over all years. For physician-dispensed medications billed at the ingredient level, mean amount paid (SD) per ingredient was $\$ 75.47$ (205.51), and when billed at the single medication level was $\$ 204.83$ (221.01). T-tests showed a mean increase in compounded medication mean amount paid between pre- and post-AB 378 groups of $\$ 12.27(P<0.001)$ for pharmacy-dispensed medications and $\$ 11.34(P<0.001)$ for physiciandispensed medications, suggesting that $A B 378$ did not curb compounded medication mean amount paid.

CONCLUSIONS: The average cost of CAWCS pharmacy- and physiciandispensed compounded medications consistently increased. Various factors may have influenced this increase, but AB 378 did not achieve its full regulatory intent to standardize billing and reimbursement and control cost. Grouping of ingredients into compounded medications allowed for application of the Colorado pricing scheme to CAWCS claims data. Adoption of Colorado pricing would save $46 \%$ of current compounded medication cost for less complicated medications, while increasing cost for more complicated medications. The analyses recommended a revised Colorado pricing scheme, which would provide improved incentives for accurate billing and lead to savings for CAWCS.

\section{J Manag Care Spec Pharm. 2019;25(7):743-51}

Copyright $\odot 2019$, Academy of Managed Care Pharmacy. All rights reserved.

\section{What is already known about this subject}

The cost of compounded medications has been rising for commercially insured patients, with the average ingredient cost increasing from $\$ 308.49$ to $\$ 710.36$ between 2012 and 2013 .

There is little evidence from clinical trials to support the use of many compound drugs.

\section{What this study adds}

This study systematically evaluated the use and cost of compounded medications, as well as billing and reimbursement patterns in a public health system setting, and found that the mean cost of compounded medications continued to rise.

Evaluating the difference in the mean cost of compounded medications suggested that as a regulation California's AB $378 \mathrm{did}$ not exert the full desired effect to curb the use and cost of compounded medications in the California Workers' Compensation System; moreover, claims that were billed incorrectly were still reimbursed.

To control compounded medication cost, an alternative pricing mechanism was suggested that would implement a more stringent, incentive-based pricing scheme, itemizing at the ingredient level but also reimbursing compounded medications as a whole rather than only paying by ingredient. 
A compounded medication is produced by "combining tailored ingredients to meet individual patient's special medical needs, providing patients with allergen-free formulations, alternative doses, combination of drugs, or dosage forms that would be otherwise commercially unavailable." 1 Pharmacies can provide compounded medications to physicians to dispense directly from their offices, or specialty pharmacies can prepare and dispense compounded medications. Although medically required in some cases, there is growing concern that compounded medications are being overprescribed, leading to questions about safety, efficacy, and necessity of the high utilization and cost. ${ }^{1}$

Previous studies have evaluated regulatory gaps in drug compounding and found that the U.S. Food and Drug Administration has increased oversight of compounding facilities to address these gaps. ${ }^{2}$ Furthermore, the increase in compounded medication prescriptions in the United States has resulted in state governments passing new laws to reign in the use of compounded medications. ${ }^{3}$ Consequently, there is a range of statutory regulations in different states that have had mixed results in controlling compounded medication use. One of these regulation plans, a tiered system where the reimbursement amount is determined by a varying number and type of ingredients and is used by the state of Colorado, is explored further in this study. ${ }^{4}$

A recent study examined the use and cost of a sample of U.S. commercially insured patients ${ }^{1}$; however, analyses on use and cost of compounded medications in noncommercially insured populations are sparse. To better understand methods to contain compounded medication use, it is important to examine use, billing, reimbursement practices, and implications of regulations and pricing mechanisms related to compounded medications in a noncommercially insured, public health system setting.

The California Workers' Compensation System (CAWCS) is the largest workers' compensation system in the United States and is a public health system that was expected to have high compounded medication use. ${ }^{5}$ However, the lack of any rules for transparency in billing for compounded medications made it difficult for CAWCS to determine the amount and reasons for the total amounts billed. As a result, California Assembly Bill \#378 (AB 378) was signed into law in 2011 and implemented on January 1, 2012, in order to increase the transparency and enable more accurate assessment of the use of compounded medications, medical foods, and convenience packages. ${ }^{6}$

Before the passage of $\mathrm{AB} 378$, it was difficult for CAWCS to assess whether certain practices were inflating the cost of compounded medications, using unnecessary ingredients, overusing ingredient quantities, overselecting the highest cost ingredients, or prescribing compounded medications when medications wete available and did not need to be compounded. AB 378 (a) requires that compounded medications must be billed at the ingredient level by a compounding pharmacy or dispensing physician, with each ingredient identified using applicable National Drug Code (NDC) numbers with the corresponding quantity and (b) prohibits separate reimbursement for ingredients with no NDC number. AB 378 also prohibited workers' compensation physicians from self-referring. Thus far, the effect of AB 378 on compounded medication use in CAWCS has not been analyzed. ${ }^{7}$

The main objectives of this study were to (a) examine use, billing, and reimbursement amounts for compounded medications at the ingredient and whole level, in order to determine the effects of CAWCS's efforts to decrease its compounded medication use, and (b) evaluate and recommend an alternative pricing mechanism that could offer incentives for appropriate use and cost control in California. More specifically, the aims for this study were as follows:

- To determine the trends in use, billing, and reimbursement of physician-dispensed and pharmacy-dispensed compounded medication prescriptions at the ingredient and single drug level in the CAWCS.

- To examine the influence of AB 378 on the use, billing, and reimbursement practices of compounded medications in CAWCS.

\section{Methods}

\section{Methods for Evaluating CAWCS Use, Billing, and Reimbursement}

Database Analysis. Compounded medication prescriptions billed at the ingredient level in CAWCS and documented in the Workers' Compensation Information System claims datasets from 2011 to 2013 were evaluated. Physician- and pharmacy-dispensed compounded medication claims were examined. Most compounded medication prescriptions adhered to California regulation and were billed at the ingredient level; however, between 2011 and 2013, 191,699 compounded medication prescriptions were billed at the compounded medication level (with multiple ingredients on 1 line and only combined cost listed for payment). Because some compounded medications were billed at the ingredient level, while others were billed at the single drug level with missing ingredient information, amounts paid (i.e., reimbursed) were analyzed separately for the 2 groups of billing practices.

Study Variables. The database contained the following variables: claim identifiers, billing identifiers, billed amount, paid (reimbursed) amounts, service adjustment codes, drug names, NDC numbers, service dates, and quantity dispensed (days covered) for each billed pharmaceutical product. Each patient in CAWCS was assigned a unique claim identifier, and each bill by pharmacy or physician was assigned a billing identifier. Service adjustment codes indicated reasons for discrepancy between billed and paid amount. For physician-dispensed 
Utilization, Cost, and Pricing Scheme of Compounded Medications for

Public Health System Patients: The California Workers' Compensation System, 2011-2013

TABLE 1 Billing and Reimbursement Pattern of Compounded Medications by Dispenser

\begin{tabular}{|c|c|c|c|c|c|}
\hline & Total Billed ${ }^{\mathrm{a}}$ & Billed by Ingredient ${ }^{b}$ & $\begin{array}{l}\text { Not Billed by } \\
\text { Ingredient }{ }^{\mathrm{c}}\end{array}$ & Reimbursed Billd & $\begin{array}{c}\text { Not Billed by Ingredient } \\
\text { but Reimbursed }\end{array}$ \\
\hline Dispensed by pharmacy & 382,719 & $324,739(85.85 \%)$ & $57,980(15.15 \%)$ & $117,570(30.72 \%)$ & $21,292(18.11 \%)$ \\
\hline 2011 & 86,862 & 71,800 & 15,062 & 32,698 & 6,552 \\
\hline 2012 & 175,034 & 147,106 & 27,928 & 55,765 & 10,722 \\
\hline 2013 & 120,823 & 105,833 & 14,990 & 29,107 & 4,018 \\
\hline Dispensed by physician & $2,219,463$ & $2,085,744(93.98 \%)$ & $133,719(6.02 \%)$ & $1,450,384(65.35 \%)$ & $101,543(7.00 \%)$ \\
\hline 2011 & 789,543 & 740,884 & 48,650 & 533,528 & 35,947 \\
\hline 2012 & 857,615 & 809,899 & 47,716 & 536,283 & 36,866 \\
\hline 2013 & 572,314 & 534,961 & 37,353 & 380,573 & 28,730 \\
\hline \multicolumn{6}{|c|}{ 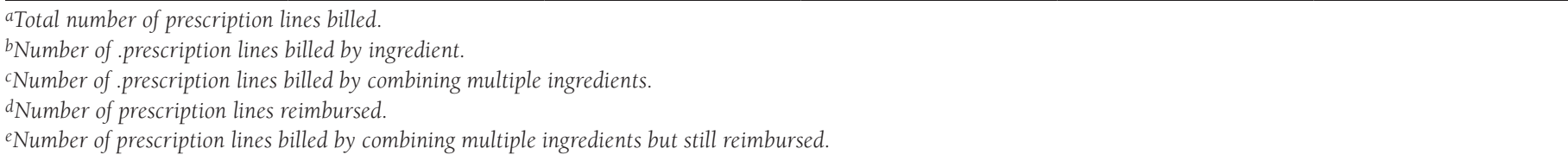 } \\
\hline
\end{tabular}

compounded medications, because 91\% of bills had missing values for the billed amount variable, analysis regarding the extent of use and cost relied on amounts reimbursed (paid) by CAWCS. For pharmacy-dispensed compounded medications, billed and reimbursed amounts were analyzed. Each pharmaceutical product was listed by drug name, NDC number, and dispensing date (service date). For the reimbursed pharmacydispensed ingredients that lacked an ingredient name or NDC number (17\% of reimbursed bills), but included terms such as "CD," "CM," and "compound," we grouped these bills with the incorrectly billed compounded medication group described in the Groupings section to enable the totals to accurately reflect all billed compounded medications.

Ingredients. Under California regulations, for reimbursement medications should be billed at the ingredient level. However, no regulation specifies that ingredients for compounded medications need to be identified as part of a single compounded medication. Thus, the claims dataset lacked a variable indicating which pharmaceutical products were part of a compounded medication. Our co-author pharmacist evaluated each line of the data and identified an ingredient as part of a compounded medication if it satisfied 1 or more of the following criteria: (a) if there was an NDC number and/or product name for a compounded medication, (b) if a product was predominantly marketed as a compounded medication ingredient, and (c) if the product bill included identified compounded medication ingredients, dosage forms, or compounding fees. A product was determined to be predominantly marketed as a compounded medication ingredient if it was cross-referenced as a compounding ingredient in a catalogue of compounding supplier sites, such as Medsica (https://www.medisca.com/) and Professional Compounding Centers of America (https:// www.pccarx.com/Products/ProductCatalog.aspx). If these criteria were satisfied, the bill was evaluated to see if there were other ingredients billed on that same date that could be part of a compounded medication. We reported ingredients, claims frequency, reimbursement rate, and total and mean paid costs. Groupings. The identified ingredients were then combined into a single compounded medication for analysis. The coauthor pharmacist identified the ingredients of the most commonly used single compounded medications from the compounded drug literature. Furthermore, a local compounding pharmacy was consulted for an ingredient list of common compounded recipes. Within a product bill for a given patient and service date, the billed ingredients were cross-referenced with the compounded recipe. Ingredients identified as commonly combined were designated as ingredients of a single compounded medication. A single compounded medication was identified by evaluating likely combinations of ingredients that were billed on the same date. If clinically appropriate, we assumed that every dosage form billed consisted of 1 compounded medication. We assumed that within a product bill, multiple line items of the same drug indicated unique compounded products. We also assumed that duplicate ingredients (with the same mechanism of action) were used to compound distinct products. For example, if Lipoderm base appeared in 3 lines, flurbiprofen appeared in 2 lines, and ketoprofen appeared in 1 line, this would indicate 3 separate compounds: Lipoderm/flurbiprofen, Lipoderm/flurbiprofen, and Lipoderm/ketoprofen. Only ingredients from the pharmacy-dispensed dataset were grouped into single compounded medications due to the high volume of physician-dispensed compounded medications.

\section{Methods for AB 378 Regulatory Evaluation}

The legislative intent of $A B 378$ was to address the expected overuse of compounded medications, ${ }^{8}$ medical foods, and convenience packages; the analysis here assessed only its effect 
Utilization, Cost, and Pricing Scheme of Compounded Medications for

Public Health System Patients: The California Workers' Compensation System, 2011-2013

TABLE 2 Descriptive Statistics of Reimbursement of Compounded Medications by Dispenser ${ }^{a}$

\begin{tabular}{|c|c|c|c|c|c|c|}
\hline & $\begin{array}{c}\text { Number of } \\
\text { Claims }\end{array}$ & $\begin{array}{l}\text { Mean Amount } \\
\text { Paid, \$ }\end{array}$ & $\begin{array}{l}\text { Standard } \\
\text { Deviation }\end{array}$ & Minimum & Lowest Amount & Maximum \\
\hline \multicolumn{7}{|c|}{ Compounded medication ingredients } \\
\hline Dispensed by pharmacy & 70,964 & 45.40 & 195.97 & 0 & .05 & $4,371.57$ \\
\hline 2011 & 17,384 & 25.78 & 141.87 & 0 & .05 & $1,654.00$ \\
\hline 2012 & 33,609 & 32.80 & 172.78 & 0 & .09 & $2,913.38$ \\
\hline 2013 & 19,971 & 83.68 & 258.16 & 0 & .09 & $4,371.57$ \\
\hline Dispensed by physician & $1,950,003$ & 75.47 & 207.51 & 0 & .02 & $28,050.00$ \\
\hline 2011 & 678,527 & 68.74 & 173.12 & 0 & .13 & $9,508.28$ \\
\hline 2012 & 768,371 & 68.90 & 210.10 & 0 & .03 & $19,616.00$ \\
\hline 2013 & 503,105 & 94.58 & 242.01 & 0 & .02 & $28,050.00$ \\
\hline \multicolumn{7}{|c|}{ Single compounded medication } \\
\hline Dispensed by pharmacy & 39,571 & 191.17 & 515.18 & 0 & .04 & $13,577.40$ \\
\hline 2011 & 13,768 & 87.11 & 289.52 & 0 & .04 & $6,865.38$ \\
\hline 2012 & 17,340 & 169.33 & 452.59 & 0 & .17 & $6,541.92$ \\
\hline 2013 & 8,463 & 419.27 & 783.84 & 0 & .30 & $13,577.40$ \\
\hline
\end{tabular}

on compounded medications. To analyze differences in cost of compounded medications before and after $\mathrm{AB} 378$, we categorized prescriptions into 2 groups: (1) the pre-AB 378 group included all compounded medication prescriptions from 2011 and (2) the post-AB 378 group included all prescriptions from 2012 and 2013. T-tests were used to assess if AB 378 was associated with a difference in the mean of compounded medication use and cost before and after $\mathrm{AB} 378$.

\section{Results}

\section{Evaluating CAWCS Use, Cost, and Reimbursement}

Billing Practices. Billing and reimbursement patterns are presented in Table 1. Despite the AB 378 requirement that compounded medications be billed at the ingredient level, of 382,719 pharmacy-dispensed compounded medication prescription lines billed, 15\% (57,980 lines) were not billed at the ingredient level. For physician-dispensed compounded medications, $6 \%$ (133,719 of 2,219,463 lines) were not billed at the ingredient level. Not all of these bills were reimbursed. For pharmacy-dispensed compounded medications, excluding the billed prescription lines denied by CAWCS-as indicated by a service adjustment code-18\% (21,292 of 117,570 lines) of reimbursed bills over all years were not billed at the ingredient level. Annually, 20\% (6,552 of 32,698 lines) of reimbursed bills in $2011 ; 19 \%$ (10,722 of 55,765 lines) in 2012; and 14\% $(4,018$ of 29,107 lines) in 2013 were not billed at the ingredient level. For physician-dispensed compounded medications, 7\% (101,543 of 1,450,384 lines) overall were not billed at the ingredient level. Annually, 7\% (35,947 of 533,528 lines) of reimbursed bills in 2011; 7\% (36,866 of 536,283 lines) in 2012; and $8 \%(28,730$ of 380,573 lines) in 2013 were not billed correctly.

Not billing at the ingredient level persisted after AB 378 required compounded medications to be billed as such for reimbursement. While the number of claims and percentage of incorrect billing decreased for pharmacy-dispensed compounded medications, the percentage of incorrect billing reimbursed by CAWCS increased slightly for physician-dispensed compounded medications, although the overall number of claims submitted declined.

Extent of Use and Cost at the Ingredient Level. Reimbursed compounded medications by CAWCS represented approximately $1,567,954$ of 20 million total prescriptions across all 3 years, or about 522,651 prescriptions annually. This use represented approximately $\$ 167$ million paid to pharmacies and physicians over this period, which was about $\$ 56$ million paid annually.

Physician-dispensed compounded medication payments were approximately $\$ 47$ million in 2011, \$58 million in 2012, and \$54 million in 2013. Pharmacy-dispensed compounded medication payments were approximately $\$ 1.2$ million in 2011, \$2.9 million in 2012, and $\$ 3.5$ million in 2013. While total amounts paid for physician-dispensed compounded medications were less in 2013 than in 2012, total amounts paid for pharmacy-dispensed compounded medications increased steadily.

For those pharmacy-dispensed compounded medications billed at the ingredient level, as shown in Table 2, mean amount paid (standard deviation [SD]) per compounded medication ingredient was $\$ 45.40$ (195.97) and for those compounded medications billed at the single medication level, mean amount paid (SD) per compounded medication was $\$ 95.20$ (326.33). Mean amount paid (SD) per ingredient rose annually from $\$ 25.78$ (141.87) in 2011, $\$ 32.80$ (172.78) in 2012, and more than tripled to $\$ 83.68$ (285.16) in 2013. Mean amount paid (SD) per single compounded medication also rose dramatically from $\$ 52.08$ (206.70) in 2011, \$99.41 (330.49) in 2012, and \$154.28 (443.49) in 2013. 
Utilization, Cost, and Pricing Scheme of Compounded Medications for

Public Health System Patients: The California Workers' Compensation System, 2011-2013

\section{TABLE 3 Most Frequently Billed Compounded Medication Ingredients}

\begin{tabular}{|c|c|c|c|c|c|}
\hline \multicolumn{3}{|c|}{ Dispensed by Pharmacy } & \multicolumn{3}{|c|}{ Dispensed by Physician } \\
\hline \multirow{2}{*}{$\begin{array}{l}\text { Most Frequently Billed Ingredients } \\
\text { Cream base }\end{array}$} & \multicolumn{2}{|c|}{ n (\%) } & \multirow{2}{*}{$\begin{array}{l}\text { Most Frequently Billed Ingredients } \\
\text { Cream base }\end{array}$} & \multicolumn{2}{|c|}{ n (\%) } \\
\hline & 61,661 & $(16.11)$ & & 385,343 & $(17.23)$ \\
\hline N/A & 57,512 & (15.03) & Cyclobenzaprine & 229,260 & (10.33) \\
\hline Capsaicin & 35,849 & $(9.37)$ & Medrox & 170,766 & $(7.69)$ \\
\hline Menthol & 29,486 & $(7.70)$ & Tramadol & 158,450 & (7.14) \\
\hline Flurbiprofen & 29,343 & $(7.67)$ & Dendracin & 154,491 & $(6.96)$ \\
\hline Camphor & 28,874 & $(7.54)$ & Terocin & 131,608 & (5.93) \\
\hline Tramadol HCL & 27,615 & $(7.22)$ & Ketoprofen & 131,325 & $(5.92)$ \\
\hline Cyclobenzaprine & 20,431 & $(5.34)$ & Flurbiprofen & 104,696 & $(4.72)$ \\
\hline Ketoprofen & 17,587 & $(4.60)$ & Menthol & 89,255 & $(4.02)$ \\
\hline Gabapentin & 12,757 & (3.33) & Amitriptyline & 73,807 & (3.33) \\
\hline Others & 61,604 & $(16.10)$ & Others & 590,462 & $(26.60)$ \\
\hline Total & 382,719 & $(100.00)$ & Total & $2,219,463$ & $(100.00)$ \\
\hline
\end{tabular}

N/A = not applicable.

For physician-dispensed compounded medications billed at the ingredient level, mean amount paid (SD) per ingredient over all years was $\$ 75.47$ (205.51) and when billed per compounded medication was $\$ 204.83$ (221.01), higher than the mean amount paid when dispensed by a pharmacy. Annually, mean amount paid (SD) per ingredient was $\$ 68.74$ (173.12) in 2011, \$68.90 (210.10) in 2012, and \$94.58 (242.01) in 2013. Mean amount paid (SD) per single compounded medication was $\$ 174.31$ (164.90) in 2011, \$185.56 (191.60) in 2012, and $\$ 266.83$ (292.23) in 2013. Mean amount paid (SD) per ingredient, as well as per single medication steadily increased from 2011 to 2013. Regardless of whether the prescription was billed correctly at the ingredient level, the average amount paid increased every year for pharmacy- and physician-dispensed compounded medications.

The most common pharmacy-dispensed ingredientsexcluding delivery agents such as cream bases (16.1\%)—were capsaicin (9.4\%), menthol (7.7\%), camphor (7.5\%), tramadol (7.2\%), cyclobenzaprine (5.3\%), ketoprofen (4.6\%), and gabapentin (3.3\%). These 7 ingredients accounted for $60 \%$ of the prescriptions. Top ingredients included agents from analgesic, local anesthetic, opioid, skeletal muscle relaxant, anti-inflammatory, and anticonvulsant pharmacologic categories (Table 3).

Top physician-dispensed ingredients were distinct from pharmacy-dispensed ingrediants and included cyclobenzaprine (10.3\%), Medrox (7.7\%), tramadol (7.1\%), Dendracin (7.0\%), Terocin (5.9\%), ketoprofen (5.9\%), flurbiprofen (4.7\%), menthol (4.0\%), and amitriptyline (3.3\%; Table 3). These 12 ingredients accounted for approximately $60 \%$ of physiciandispensed ingredients, with 15\% of additional ingredients that were delivery agents. Top ingredients included skeletal muscle relaxants, analgesics, opioids, anti-inflammatories, and antidepressants pharmacologic categories (Table 3).
Cost by Single Compounded Medications (Grouped by Ingredient Level). We examined costs of single medications for pharmacy-dispensed drugs, in addition to the more common ingredient-level analysis found in the literature (Table 2). Mean amount paid (SD) per single compounded medication from 2011 to 2013 was $\$ 194.17$ (515.18). Yearly, mean amount paid (SD) per medication in 2011 was $\$ 87.11$ (289.52), \$169.33 (452.59) in 2012, and $\$ 419.27$ (783.84) in 2013, which was a dramatic increase even after the implementation of $A B 378$ (Table 4).

\section{AB 378 Regulatory Evaluation}

For pharmacy- and physician-dispensed compounded medications and ingredients, t-tests suggest that $\mathrm{AB} 378$ did not curb the cost of compounded medications (Table 4). For pharmacydispensed prescriptions, the pre- $\mathrm{AB} 378$ group included 32,698 compounded medication and ingredient prescriptions. The post-AB 378 group included 84,872 compounded medication and ingredient prescriptions. For physician-dispensed prescriptions, the pre-AB 378 group included 469,404 compounded medication and ingredient prescriptions. The postAB 378 group included 842,058 compounded medication and ingredient prescriptions.

The mean increase in total pharmacy-dispensed compounded medication costs between the pre-AB 378 group year and post-AB 378 group years was $\$ 12.27(P<0.001)$ and $\$ 11.34$ $(P<0.001)$ for physician-dispensed compounded medication costs. This result suggests that AB 378 did not curb costs; rather, the costs increased.

\section{Alternative Pricing Mechanism}

Given that the average yearly cost of CM in CAWCS was $\$ 56$ million and the absence of a clear effect from $\mathrm{AB} 378$, pricing mechanisms in other states were surveyed to provide a 
Utilization, Cost, and Pricing Scheme of Compounded Medications for

Public Health System Patients: The California Workers' Compensation System, 2011-2013

TABLE 4 T-test Evaluation of the Effect of AB 378 on Cost of Each Billed Prescription Line

\begin{tabular}{|c|c|c|c|c|c|c|c|c|}
\hline & & $\begin{array}{c}\text { Number of } \\
\text { Claims }\end{array}$ & $\begin{array}{l}\text { Mean Amount } \\
\text { Paid, \$ }\end{array}$ & $\begin{array}{l}\text { Standard } \\
\text { Deviation }\end{array}$ & $\mathrm{t}$ & Df & $P$ Value & $\begin{array}{c}\text { Mean } \\
\text { Difference, \$ }\end{array}$ \\
\hline \multirow{3}{*}{$\begin{array}{l}\text { Dispensed by } \\
\text { pharmacy }\end{array}$} & Before AB 378 & 32,698 & 16.99 & 114.98 & & & & \\
\hline & & & & & -20.03 & 117,568 & $<0.001$ & 12.27 \\
\hline & After AB 378 & 84,872 & 29.27 & 169.76 & & & & \\
\hline \multirow{3}{*}{$\begin{array}{l}\text { Dispensed by } \\
\text { physician }\end{array}$} & Before AB 378 & 469,404 & 73.44 & 173.37 & & & & \\
\hline & & & & & -37.36 & $1,311,460$ & $<0.001$ & 11.34 \\
\hline & After AB 378 & 842,058 & 84.78 & 225.37 & & & & \\
\hline
\end{tabular}

cost control alternative. Most states based their compounding drug pricing scheme on a variation of average wholesale price (AWP). For example, Louisiana's formula for reimbursement is AWP $+40 \%+$ dispensing fee at the ingredient level, and North Dakota uses AWP-72\% + dispensing fee at the ingredient level. Similarly, Ohio uses AWP (of the commonly stocked package size) $-9 \%$ for each ingredient. These reimbursement formulae reimburse more per ingredient than the current CAWCS reimbursement policy, which limits reimbursement of pharmacydispensed compounded medications to $100 \%$ of Medi-Cal's (Medicaid) system rate per ingredient and limits physician-dispensed compounded medication reimbursement to total cost for the compounded cost at 300\% (and no more than \$20) of the documented paid costs. By decreasing the reimbursement percentage, these AWP-based formulae may reduce the cost per ingredient when compared with CAWCS pricing and likely would provide more cost control than the current CAWCS system. However, while modifying a reimbursement rate to a lower percentage may reduce the cost of each ingredient, it may also incentivize prescribers to include more ingredients in each compounded medication. Following this logic, a different pricing mechanism and its potential for increasing overall cost control of compounded medications for CAWCS was evaluated.

The Colorado Pricing Scheme. The alternative mechanism recommended here is based on the state of Colorado's reimbursement scheme-a tier model in which categories of compounded medications, which vary by number and type of ingredient, determines the reimbursement amount. ${ }^{9}$ There are 4 categories of compounded medications in the Colorado system. Category I is priced at $\$ 75$ and includes anti-inflammatory medications or local anesthetic single agents. Category II is priced at $\$ 150$ and includes anti-inflammatory agents or agents in combination with a local anesthetic agent or agents. Category III is priced at $\$ 250$ and includes a single agent other than an anti-inflammatory agent or local anesthetic, either alone or in combination with anti-inflammatory or local anesthetic agents. Category IV is priced at $\$ 350$ and includes 2 or more agents that are not anti-inflammatory or local anesthetic agents, either alone or in combination with other anti-inflammatory or local anesthetic agents. In this scheme, category fees include materials, shipping, handling, and time. All ingredients must be listed by quantity used per prescription. Regardless of how many ingredients or what type, compounded medications cannot be reimbursed higher than the Category IV fee. This scheme provides a better incentive structure than CAWCS's current pricing system.

Applying the Colorado Reimbursement Scheme. To evaluate whether the Colorado pricing scheme would control costs more than the current CAWCS pricing system requires that ingredients be grouped into specific compounded medications. Using groupings from CAWCS pharmacy-dispensed claims, a pharmacist recoded each compounded medication into 1 of 4 Colorado pricing categories. Any medication that did not fit into 1 of the categories was priced at $\$ 0$ because, under Colorado pricing, medications that did not fit into any of the 4 categories would not be reimbursed. The total cost of CAWCS pharmacy-dispensed compounded medications using Colorado pricing was then calculated.

Table 5 compares CAWCS and Colorado pricing. Average cost per compounded medication was $\$ 267.10$ under Colorado pricing, which was higher than the CAWCS pricing of $\$ 238.66$. However, the average cost per compounded medication was lower for medications in Category I (\$75 compared with \$246) and Category II (\$150 compared with \$184.37) under Colorado pricing than under CAWCS pricing. If CAWCS were to simply adopt the Colorado pricing scheme for Category I and Category II compounded medications, CAWCS would save $46 \%$ of the current cost of Category I and Category II medications.

On the other hand, the average cost per compounded medication was higher for medications in Category III ( $\$ 250$ compared with \$195.86) and Category IV (\$350 compared with \$284.28) under Colorado pricing than under CAWCS pricing. Total cost was lower for compounded medications in Category I and Category II under Colorado pricing than under CAWCS pricing, while total cost was higher for Category III and Category IV medications under Colorado pricing than under CAWCS pricing. The analysis suggested that using the refinement of the Colorado pricing system in California could lead to savings for CAWCS. 
Utilization, Cost, and Pricing Scheme of Compounded Medications for

Public Health System Patients: The California Workers' Compensation System, 2011-2013

TABLE 5 CAWCS Pharmacy-Dispensed Compounded Medication Pricing and Colorado Pricing

\begin{tabular}{|c|c|c|c|c|}
\hline & $\begin{array}{c}\text { Grouped Compounded } \\
\text { Medications } \mathrm{s}^{\mathrm{a}} \\
\mathrm{n}\end{array}$ & $\begin{array}{c}\text { CAWCS } \\
\text { Paid Amount } \\
\$\end{array}$ & $\begin{array}{c}\text { Colorado Alternative } \\
\text { Pricing } \\
\$\end{array}$ & $\begin{array}{c}\text { Modified Colorado } \\
\text { Pricing } \\
\$\end{array}$ \\
\hline Average cost per medication & 39,571 & 239 & 267 & 194 \\
\hline Total cost & & $7,666,641$ & $8,598,950$ & $6,222,150$ \\
\hline \multicolumn{5}{|l|}{ By category } \\
\hline $\mathrm{I}$ & 4,042 & 994,342 & 303,150 & 303,150 \\
\hline II & 726 & 122,853 & 108,900 & 108,900 \\
\hline III & 13,926 & $2,727,538$ & $3,481,500$ & $2,785,200$ \\
\hline IV & 13,444 & $3,821,909$ & $4,705,400$ & $3,024,900$ \\
\hline \multicolumn{5}{|c|}{ Average cost per medication by year } \\
\hline 2011 & 13,768 & 87 & 117 & 176 \\
\hline 2012 & 17,340 & 169 & 201 & 200 \\
\hline 2013 & 8,463 & 419 & 486 & 206 \\
\hline \multicolumn{5}{|c|}{ Average cost per medication by category } \\
\hline $\mathrm{I}$ & 4,042 & 246 & 75 & 75 \\
\hline II & 726 & 184 & 150 & 150 \\
\hline III & 13,926 & 196 & 250 & 200 \\
\hline IV & 13,444 & 284 & 350 & $\begin{array}{c}\text { removed } \\
\text { (estimated average \$225.00) }\end{array}$ \\
\hline
\end{tabular}

Refinement of the Colorado Pricing Scheme. Simply using Colorado pricing for Category I and Category II medications would lower cost for CAWCS. However, compounded medications with more ingredients, which would place them in Categories III and IV, would require a revised system that would include repricing and recategorization. One way to refine the system would be to lower the reimbursement amount to $\$ 200$ for Category III and remove Category IV. If there were more than 2 agents in a compounded medication, they should be billed separately using Category I pricing of $\$ 75$ per antiinflammatory medication or local anesthetic single agent and Category II pricing of $\$ 150$ when the medication included an anti-inflammatory agent in combination with a local anesthetic agent. The possible cost for CAWCS compounded medications that were grouped in Category IV would be $\$ 150, \$ 225$, or $\$ 300$ instead. The average cost thus would be $\$ 225$.

In the revised pricing scheme, costs of Category I and Category II compounded medications would be consistent with the current Colorado pricing system. Compounded medications in Category III would have a total cost of $\$ 2,785,200$ and am average cost of $\$ 200$. Medications in Category IV would have a total cost of $\$ 3,024,900$ and an average cost of $\$ 225$. In total, a revised Colorado pricing scheme would reduce the cost of compounded medications from 2011 to 2013 in CAWCS by approximately $\$ 1.4$ million-reduction from $\$ 7,666,641$ to $\$ 6,222,150$ - and provide more control over "ingredientcreep," where additional, often unnecessary ingredients are included into a compounded medication for the purpose of increasing the payment.

\section{Discussion}

CAWCS claims were evaluated to shed light on current use, billing, and reimbursement practices of compounded medications in a noncommercially insured health system setting. Compounded medications were analyzed at the ingredient level and then grouped into pharmacy-dispensed single compounded medications. This grouping provided a unique variable for analysis and allowed for evaluation of an alternative pricing mechanism, which priced compounded medications by categories, and its cost-controlling potential in California. This study's grouping methodology derived from McPherson et al.'s methodology (2016), ${ }^{1}$ which adopted a data mining technique with a priori algorithms for compounded medication identification-grouping medications by the frequency in which they are compounded together. This study evaluated each bill, which may contain a long list of compounded medication ingredients, by taking into consideration active ingredients in a claims bill, dosage forms, quantity of ingredients, and cost of ingredients.

The analyses showed that frequency of prescriptions for compounded medication ingredients and single medications in pharmacy- and physician-dispensed data fluctuated throughout the study period. However, the mean amount paid consistently increased over time for pharmacy- and physician-dispensed compounded medications. Mean amounts paid increased for both types of prescriptions, which were billed correctly at the ingredient level and at the single drug level. 
Study results supported previously reported trends of increasing compounded medication costs across states and workers' compensation systems. For example, Ireland and Swedlow (2010) found that payment for medical foods, convenience packages, and compounded medications grew from $2.3 \%$ to $12.0 \%$ of medication expenses between 2006 and 2009. ${ }^{3}$ Wynn (2011) concurred and showed that some parties faced significant financial incentives to promote use of compounded medications. ${ }^{8}$ As such, it is crucial to regulate use and pricing of compounded medications.

While various factors can influence the cost of compounded medications, AB 378 may not have achieved its full regulatory intent to reign in these costs, as demonstrated by the increase in mean costs between pre- and post- $A B 378$ for pharmacy- and physician-dispensed compounded medication costs. Rising costs is a persistent issue that has not been remedied by new regulations, which also have not been consistently enforced. We showed that compounded medications were not billed consistently by ingredient but were reimbursed despite incorrect billing. Lack of enforcement may weaken the effect of regulations, since providers may be less inclined to adjust their billing practices to adhere to regulations. Current regulations were also unable to change the incentive structure for prescribing compounded medications with high numbers of ingredients, which accounted for higher costs in 2013. We suggest that CAWCS should consider a new pricing mechanism for controlling these practices of seemingly legitimate billing by increasing the number of ingredients in compounded medications. The Colorado pricing scheme is a system that might provide control over potential ingredient creep.

All of the most common agents in pharmacy- and physiciandispensed compounded medications included pharmacologic agents used to treat somatic and neuropathic pain, as would be expected for a workers' compensation system. However, most common ingredients used shed light on differences in mean amount paid per ingredient between pharmacy- and physician-dispensed compounded medications. Among pharmacy-dispensed claims, top ingredients billed were listed at the ingredient level, consistent with AB 378 intent. On the other hand, physician-dispensed claims listed commercially available combination products (e.g., Medrox [methyl salicylate 20\%/menthol 5\%/capsaicin 0.0375\%], Dendracin [methyl salicylate 30\%/capsaicin 0.0375\%/menthol 10\%], and Terocin [lidocaine $4 \% /$ menthol $4 \%$ ]) as starting ingredients in their compounded medications. Using these combination products, which are available over the counter, may be a driver in the doubling of the mean amount paid per compounded medications billed at the ingredient level for physician-dispensed medications compared with pharmacy-dispensed medications. Current reimbursement mechanisms may not provide consistent incentive to use the least costly starting materials across pharmacies and dispensing physician offices.
Comparison of yearly CAWCS compounded medication cost with Colorado pricing further suggested that the prescription of compounded medications is becoming increasingly complicated; more and more ingredients are being included in each medication. The average cost using Colorado pricing was \$116.51 in 2011, \$201.18 in 2012, and \$485.54 in 2013 . Increase in average yearly prices showed that more and more compounded medications are those with more than 1 agent and therefore would be billed in higher priced categories. This finding further highlighted the need of a new pricing mechanism.

The pattern of cost control using a revised Colorado scheme also could apply to physician-dispensed compounded medications. Given that physician-dispensed medications shared the same complicated combination of ingredients as pharmacydispensed medications, the revised Colorado pricing would lead to substantial reduction in cost for CAWCS. CAWCS recognizes the importance of billing by ingredients and also which ingredients were grouped together as a compounded medication. Therefore, since 2016, CAWCS started to collect that information in its billing system. Unfortunately, the data lag means that complete claims data would not be available for analysis until later. This variable is essential for CAWCS to transition into a different pricing mechanism.

\section{Limitations}

This study has some limitations to consider, some of which are inherent in the use of claims data. First, while the CAWCS data available for this study covered the years from 2011 to 2015, only data from 2011 to 2013 were analyzed because data from 2014 to 2015 were incomplete. Claims data can take 6 months to 1 year or longer to be fully resolved, and the low volume of accounts observed suggested incomplete data.

Second, as with many claims datasets, there were missing data, duplications, and potential claims errors. The service adjustment code was used to identify duplications and errors and to decide if each empty cell was empty because of missing data or was simply a zero for billed, reimbursed (paid), denied, or adjusted amounts. Compounded medications claims consisted of multiple drug elements, increasing the chance for errors in claim entries. We have outlined in the Methods section how we evaluated each of these claims to most accurately categorize the compounded ingredients and medications. However, we recognize the importance for future studies to continue evaluating compound drug prescription and billing patterns.

Finally, this analysis is only the first step in examining the full regulatory effect of $A B$ 378. T-tests used to examine the difference in cost before and after $\mathrm{AB} 378$ do not account for confounding variables. This study was unable to control other factors influencing cost because of the limitation of available variables of interest in the dataset. It would be beneficial for future analyses to control for these factors. 


\section{Utilization, Cost, and Pricing Scheme of Compounded Medications for Public Health System Patients: The California Workers' Compensation System, 2011-2013}

Despite these limitations, this study shed light on the use and cost of compounded medications in a public health system. Furthermore, the analyses evaluated and compared the cost of compounded medication under a different pricing scheme.

\section{Conclusions}

CAWCS is one of the health systems experiencing high compounded medication costs. However, regulations aiming to contain these costs have been unsuccessful. ${ }^{6}$ To identify a way to contain costs, this study examined compounded medication use, billing, reimbursement pattern, implication of related regulations, and how different pricing mechanisms could alter reimbursement costs in the context of CAWCS.

The analysis found that mean amount paid per compounded ingredients and single compounded medications consistently increased for pharmacy- and physician-dispensed medications. Various factors can influence cost, but the regulation AB 378 did not achieve its full regulatory intent to reign in cost. We also found that the requirement to bill at the ingredient level was not being followed, and we suggest that payment should only be made if these requirements are followed.

Our unique dataset grouped ingredients into compounded medications, which allowed for the application of Colorado's pricing scheme to CAWCS claims data. The results suggested that CAWCS required a refinement of this system for cost containment; therefore, a revised pricing system was proposed in which pricing for Category I and Category II compounded medications remained the same as Colorado pricing. The cost of Category III pricing was lowered from $\$ 250$ to $\$ 200$ per medication. Category IV was removed, and medications in Category IV were simplified into Category I and Category II medications. This revision resulted in an average cost of $\$ 225$ per compounded medication for those drugs that were previously in Category IV and was not expected to affect patient access to compounded medications. While Colorado pricing could lead to cost control, it is important to note that in a system where fixed pricing is used for reimbursement there may be bureaucratic cost when making market adjustments for inflation of prices over time. However, the revised pricing system should lead to cost control for pharmacy-dispensed medications in CAWCS and the pattern of savings should also apply to physician-dispensed medications.

There are various factors at play when it comes to reimbursing compounded medication prescriptions. One method to control costs is to implement a more stringent, incentive-based pricing scheme that itemizes at the ingredient level but that also reimburses costs as a whole rather than only paying by ingredient. Our revised pricing plan demonstrated substantial potential for cost control if used by CAWCS.

\section{Authors}

TRACY KUO LIN, MSc, PhD, and LESLIE WILSON, PhD, University of California, San Francisco. DANG TRAN, PharmD, Chinese Community Health Plan, San Francisco, California, and OSAMA A. SHOAIR, PhD, The University of Texas at Tyler.

AUTHOR CORRESPONDENCE: Tracy Kuo Lin, MSc, PhD, University of California, 3333 California St., Ste. 350-A, San Francisco, CA 94118. Tel.: 425.753.1879; E-mail: tracy.lin@ucsf.edu.

\section{DISCLOSURES}

Funding for this study was provided by the California Workers' Compensation System. The authors had final control regarding study design, study conduct, and writing of the manuscript. The authors report no conflicts of interest.

\section{REFERENCES}

1. McPherson T, Fontane P, lyengar R, Nederson R. Utilization and costs of compounded medications for commercially insured patients, 2012-2013. J Manag Care Spec Pharm. 2016;22(2):172-81. Available at: https://www.jmcp. org/doi/10.18553/jmcp.2016.22.2.172

2. Shepherd J. Regulatory gaps in drug compounding: implications for patient safety, innovation, and fraud. DePaul Law Review. Forthcoming. Posted on SSRN. June 5, 2018. Available at: https://papers.ssrn.com/sol3/ papers.cfm?abstract_id=3182087. Accessed May 14, 2019.

3. Ireland J, Swedlow A. The cost and utilization of compound drugs, convenience packs, and medical foods in California workers' compensation. CWCI Research Notes. August 2010. Available at: https://www.cwci.org/ document.php?file=1337.pdf. Accessed May 14, 2019.

4. Walls AP, Johnson S, Nguyen M, O'Lenic K, Pokorney T, Randolph S. Compounding is confounding workers' compensation. CompPharma. 2014. Available at: https://comppharma.com/wp-content/uploads/2016/09/ CompoundDrugResearch.pdf. Accessed May 14, 2019.

5. Safety National. California WC system: a regulator's perspective. Conference Chronicles. December 14, 2015. Available at: https://www. safetynational.com/conferencechronicles/california-wc-system-a-regulatorsperspective/. Accessed May 14, 2019

6. California State Assembly. California Assembly Bill 378. 2011. Available at: http://www.leginfo.ca.gov/pub/11-12/bill/asm/ab_0351-0400/ab_378_ bill_20110509_amended_asm_v97.html. Accessed May 14, 2019.

7. Swedlow A, Auen E. Current trends in compound drug utilization and cost in the California Workers' Compensation System. CWCI Research Notes. February 2013. Available at: https://www.cwci.org/document. php?file=1815.pdf. Accessed May 14, 2019.

8. Wynn BO. Use of compound drugs, medical foods, and co-packs in California's workers' compensation program: an overview of the issues. WR-828-CHSWC. January 2011. Available at: https://www.dir.ca.gov/chswc/ Reports/2011/CHSWC_UseofCompoundDrugsMedicalFoodsCo-Packs.pdf. Accessed May 14, 2019.

9. Code of Colorado Regulations. Workers' compensation rules of procedure with treatment guidelines. 2016. Available at: https://www.sos.state.co.us/ CCR/GenerateRulePdf.do? ruleVersionId $=6566 \&$ fileName $=7 \% 20 \mathrm{CCR} \% 20$ 1101-3\%20Rule\%2018. Accessed June 5, 2019. 